\title{
VARIATION OF THE EXPLOSION RISK IN A HYBRID COLLECTOR DURING REVAMPING OPERATIONS
}

\author{
S. COPELLI ${ }^{1}$, M. RABONI ${ }^{2}$, M. RAGAZZI ${ }^{3}$, E. C. RADA ${ }^{3}$, V. TORRETTA ${ }^{4}$ \\ ${ }^{1}$ Department of Science and High Technology, Insubria University, Italy. \\ ${ }^{2}$ Department of Industrial Engineering, Carlo Cattaneo University, Italy. \\ ${ }^{3}$ Department of Civil, Environmental and Mechanical Engineering, University of Trento, Italy. \\ ${ }^{4}$ Department of Theoretical and Applied Sciences, Insubria University, Italy.
}

\begin{abstract}
Dusts removal from gaseous streams is a very common operation carried out in a number of industrial plants, e.g. those ones dedicated to the manufacturing of cement and steel, in order to meet the more increasingly stringent law requirements. Therefore, in the last decades, such industries have been to face more and more often the necessity to improve their de-dusting systems inspired by the principle of the 'maximum safety technologically feasible', but also pushed by the increased penetration of environmental issues into the public opinion. Unfortunately, the budget dedicated for all these improvements is often very low because de-dusting is, in the major part of the practical cases, an operation having a low influence on production performances; therefore, it is seen more as a 'necessary cost' than a profit generator. The unavoidable consequence is that plant managers investments are dedicated to other more fruitful processes than de-dusting, addressing the low budget available for gas stream purification to the socalled revamping strategies: that is, the reuse of existing de-dusting plants, either by enhancing their efficiencies through the use of various technical tricks or by adding another pieces of equipment, rather than install new and more technologically advanced plants. This solution is considered very interesting in terms of profit since the costs for the decommissioning of the obsolete equipments does not exist (in fact the old plant remains). Obviously, all the economic benefits derived from these strategies are counterbalanced by a series of technical disadvantages. In particular, a systematic risk assessment of safety of the whole new configuration of the revamped plant is necessary. In fact, such an evaluation cannot be limited to a safe design of the new installed equipment, but it has to be extended to the already existing equipment also considering the impact that changes in process conditions (induced by the new equipment) can have on the whole plant.

In this work, the relevant problem of an explosion in the de-dusting section of a cement plant, due to the establishment of an overpressure inside the apparatus, has been considered. Particularly, using fault tree analysis, it has been evaluated the changing in the overall risk (considering, for simplicity, 1 year of mission time) of explosion referring to a hybrid-like collector realized by introducing a Fabric Filter (FF) downstream with respect to an Electrostatic Precipitator (ESP). Results have shown that a chain of failures in the FF section may affect relevantly the explosion risk in the collector leading to the unavoidable need for the introduction of mitigation actions into the system.
\end{abstract}

Keywords: cement plants, explosions, hybrid collectors, revamping, risk assessment, safety.

\section{INTRODUCTION}

One of the most important mineral industries in the world, accounting only in the United States for an annual production capacity greater than 73 million tons, is cement manufacturing [1]. In Europe, this industry is not so developed and, during the last years, a relevant decrease in the consumption of cement has been registered. As the sake of example, in 2012, there has been a decrease in the manufacturing of cement of about $22 \%$ (with respect to the previous year) and this negative trend is actually continuing [2].

As a general consideration, cement industry is very capital intensive. Energy costs can account for up to $40 \%$ of the total cost of cement manufacturing. Currently, over $90 \%$ of the installed capacity uses coal as primary fuel for the kiln burner. Unfortunately, the use of coal as fuel is very dangerous because of the possible triggering of fire and explosions during cement 
manufacturing. In the last years, an innovative solution was proposed: the use of the Solid Recovered Fuel obtained from Municipal Solid Waste in order to substitute a big part of the coal [3, 4].

Cement plants are recognized as a source of undesirable airborne emissions. In an attempt to moderate the effects of such compounds on the environment and neighboring population, governments legislate maximum permissible limits for a multitude of emission types (such as sulfur dioxide, nitrogen oxides, mercury and dusts/particulate matter).

Focusing on dust emissions, which have become in the last years the most studied problem in terms of urban pollution (independently of the source: industrial processes, traffic or cooking or domestic heating), it is possible to notice that they are not as frequently regulated as $\mathrm{SO}_{2}$, $\mathrm{NO}_{\mathrm{x}}$ or mercury [5-8]. Anyway, the most stringent regulations covered are in the EU, specifically in Germany and Austria.

Dust removal from a gas stream is a common and quite cheap operation required in many industrial processes but, in the last decades, more and more stringent law requirements have forced this common operation to become very expensive. Budgets for introducing improvements (or new units) in the already existing de-dusting equipment play a fundamental role in the general asset management strategy: in fact, de-dusting, being often an operation having low influence on production performances, is unavoidably seen more as a 'necessary cost' than a profit generator. Companies' trend, especially in the actual economic scenario, is to reuse existing de-dusting plants, enhancing their efficiencies rather than install new plants that would involve costs for the decommissioning of obsolete equipments. Unfortunately, the obvious economic benefits of retrofits are counterbalanced by technical disadvantages and possible safety-related problems. In particular, a systematic assessment of safety issues, not limited to the safety-oriented design of new machines, but extended in the evaluation of the impact that changes in process conditions (induced by the new equipments) can have on the existing ones, must be done.

Cement plants usually employ two main dust and particulate matter abatement technologies to help them meet emissions limits: ElectroStatic Precipitators (ESP) and Fabric Filters (FF). ESPs use an induced electrostatic charge to attract dust to charged surfaces. While efficient on start-up and mechanically reliable, ESPs become less effective as dust builds up on the charged surfaces, causing time lost to maintenance and cleaning and potentially causing emissions to rise above permitted levels. Fabric Filters use natural or synthetic fiber-based bags to physically remove dust from the gas-stream. With typically 4 to 12 bags working in parallel, baghouses maintain steady operation for longer than ESPs, although care must be taken with respect to the potential for damaged bags. If a bag breaks mid-operation, the potential exists for a massive emission of dust. This can have severe adverse effects for local residents, water courses and may involve financial penalties for the producer.

In this work, the relevant problem of the triggering of an explosion in the de-dusting section of a cement plant has been analyzed. Particularly, it has been calculated the changing in the overall risk of explosion (considering 1 year of mission time for the plant) referring to a relevant re-vamping case study where a hybrid-like collector has been realized by introducing a Fabric Filter downstream with respect to an already installed ElectroStatic Precipitator. The results of the analysis have shown that failures in the FF section may affect relevantly the risk of an explosion occurrence in the collector itself leading to the unavoidable need for the introduction of mitigation actions into the system.

\section{DESCRIPTION OF THE HYBRID-LIKE COLLECTOR}

Hybrid collectors are abatement systems that have been designed specifically to take into account all the factors affecting their operational efficiency. Because of this fact, they have a 
very high collection efficiency $[9,10]$, and they represent the best technology currently available in order to fully comply with the limit values laid down by specific authorizations issued by the competent bodies.

The hybrid collection system treated in this case study has been realized by a retrofit operation consisting of an addition of a fabric filter downstream an already installed electrostatic precipitation section.

Given the particular system design, the amount of dust emitted into the atmosphere is considerably lower than that allowed by the strict environmental standards in force.

Hot gases (about $300^{\circ} \mathrm{C}$ ), coming from the pre-heating section of the kiln, are partly used to dry and ventilate both the coal and the crude feed mills but, while the gases of the coal mill are filtered independently, the dusty gases used in the kiln are mixed with the gas coming from the pre-heater in a tower (called 'conditioner') where they are cooled (till about $160^{\circ} \mathrm{C}$ ) and humidified with water spray; the amount of dosed water is regulated by a computer as a function of the gas temperature.

Then, the conditioned gases enter the electrostatic section of the hybrid filter, whose design is such as to allow a very low-gas velocity (in order to favor the separation of the dusts from the gaseous stream). The operating principle of an electrostatic precipitator is based on the effect of ionization of a neutral gas (corona effect) when it passes through a strong electric field formed by issue (negative polarity) and precipitation (or collector) electrodes (positive polarity) [11]. Thanks to the very high applied voltage (in this case, $65 \mathrm{kV}$ ), the issue electrodes emit electrons by photoelectric effect and charge the dust particles of which the gas to be de-dusted is rich; in this way, the charged dust particles will be attracted by the precipitation electrodes (or plates) [12]. The dust settles on the plates so that, periodically, they have to be 'shocked' in order to be kept clean and fully operative. Particularly, an electrostatic precipitator can remove dust particles as small as $1 \mu \mathrm{m}$ with an efficiency exceeding $99 \%[13,14]$.

Immediately then, the partially de-dusted gases pass through another section: the fabric filter.

A fan located downstream of the fabric filter aspires the air flow to be de-dusted, causing its entry in the lower part of the filter; hence, the gas going up meets the rows of bags and passes from the outside to the inside of their tissue cylinder.

The particles suspended in the gas are retained on the outside of the bags thanks to both the structure of the fabric fibers and a layer of particulate laying outside the fabric of each bag (which is capable of retaining even the finer particles).

The collapse of each bag on itself, given by the outside-inside air flow, is prevented by a basket that keeps roughly the shape of a tube.

The de-dusted air inside of each bag is now aspired and removed by the filter in the upper part of the chamber to be sent to the chimney, always thanks to the fan cited above. Approximately, every $8 \mathrm{~min}$, in order to avoid the clogging of the filtering surface because of an excessive deposit of dusts, an air jet at high speed is blown inside of each bag: such a jet, impacting against the filtering surface, causes a sudden expansion of the bag and allows for the detaching of the crust of dust that has been formed on it. This cleaning system is called 'pulsed jet' and has the advantage of not having to exclude the bags from the filtration system during cleaning operations; the air jet is provided instantly through solenoid valves from dedicated storage tanks, whose operating pressure is about 6 bar, and acquires a high speed through the venturi ducts placed on the basket of each bag. To maintain continuous filtration during the cleaning operation, the air is fed to a row of bags at a time, allowing the other rows to continue to carry out their activity. The detached material falls into the hoppers at the bottom 


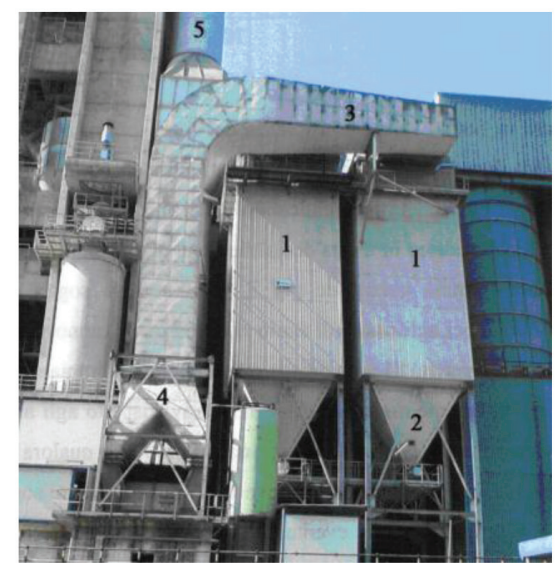

Figure 1: Sketch of the case study hybrid collector. (1) Final sections of the fabric filters; (2) hoppers; (3) de-dusted air duct; (4) fan; (5) bottom of the chimney. The ESPs are located behind the fabric filters.

of the filtering chamber and, then, it is extracted by means of a worm screw and returned to the kiln by means of conveyor belts.

All these constitutive parts of the plant have been reported in Fig. 1, which shows a simple sketch of the de-dusting unit.

\section{RISK ASSESSMENT IN CEMENT PLANTS}

\subsection{General risk assessment procedure}

When talking about Quantitative Risk Analysis (QRA), we referred to as a set of methodologies and structured procedures having the aim of determining (both qualification and quantification) the so-called 'risk function', which is a fundamental tool in a decision-making process [15].

The estimate of the risk arising from the exercise of a particular industrial activity is generally performed with a methodology that, starting from statistical data derived from the history of accidents actually occurred in plants similar to the one under study, will complete a series of specific analyses, related to a particular facility and the site where it is installed, in order to obtain a sufficiently accurate estimate of the risk [16]. In the case of mature and widespread technologies, the statistics of accidents can be so broad as to allow the direct realistic estimate of the risk or, at least, the probability of occurrence of conceivable accidents. The determination of the consequences depends very much on the site, for what concerns the aspects of meteorology and hydrology, population distribution, etc; therefore, it is generally necessary an adaptation of the statistical data to the particular case study. If you do not have a statistic sufficiently complete to be referred to, an analytical methodology to perform a risk assessment on a theoretical basis can be employed.

The risk in the performance of an industrial activity results from the use of potentially hazardous materials. The starting point of a risk analysis is always the identification of hazardous substances and the process that they undergo in the system under study. This procedure is usually referred to as hazard identification. 
The protection system (more or less sophisticated) of the plant comes into play at this point, to prevent or, at least, limit the possible effects of an accidental event. Therefore, the adverse event occurs only if you have, at the same time, the failure of the process system and the failure of the protections that the system provided to confine the incident.

Considering the physical and chemical phenomena involved in an accident, you are able to evaluate their effect on individuals, economic systems, social systems, etc.., through:

(a) an identification of the events that contribute to the risk;

(b) an estimate of the probability of occurrence of such events and their consequences;

(c) a determination of the risk function and its use for decision making.

To develop such an analysis, a thorough knowledge of the system and the industrial process implemented in it has to be acquired for the search of those failures which may constitute the source of accidents (initiating events).

The risk analyst must then develop a model of the system that allows the identification of the possible states of the latter arising from each initiating event. To estimate the probability of occurrence of each state of the system, we can make use of appropriate techniques such as fault tree analysis.

The next step is the determination of all accidental scenarios associated with each degraded state of the system (sequence of events), possibly 'filtered' by the effect of the available protection systems.

At this point, in order to identify the consequences for each category of accidents, it is necessary to develop an environmental impact model, which describes the magnitude of the consequences of each scenario. In this phase, the possible implementation of an external emergency plan can take a decisive role for the mitigation of the consequences of the accident.

Finally, after determining the probability of occurrence of each accidental event, the integration of all results, (defined as an estimate of the magnitude and frequency of occurrence) on the entire spectrum of the categories of accidental scenarios, allows for the determination of the function associated with the risk linked to the activity in question [17]. Normally, on the basis of this risk function, decision-makers (political or technical, depending on the stage at which the study is actually applied) take the appropriate decisions.

\subsection{Fault tree analysis for QRA [18, 19]}

Fault tree analysis is a technique suitable for both determining the credible modes of occurrence of an undesired event (called, top event), caused by a complex concatenation of other events (qualitative analysis), and estimating the frequency of occurrence of undesired event on the basis of frequency of occurrence of the events that cause it (quantitative analysis). It is a deductive methodology particularly suitable for the analysis of complex systems whose development can be easily decomposed into a succession of more simple events, and therefore it is well suited to the analysis of industrial installations.

Once all the systems (and their parts) have been identified, it is necessary to continue the analysis of the chain of systems, subsystems, equipment, etc., up to the failure of the individual components for which we are in possession of sufficient information on the probabilities of failure to be used in the evaluation.

These are combined through logical operations (and / or), also called logic gates, going from bottom to up, until you get to determine the probability of the top event. The fault tree 
analysis allows you to treat the contribution to risk arising from human error, as well as other causes of failure common to several subsystems. Clearly, it is possible to use fault trees also for qualitative analysis. In this case, there is the advantage of highlighting the main causes of an accident (e.g. whether failures of equipment or human error) so that you can focus on preventive measures to reduce the probability of the accident itself.

The representation of a fault tree uses some symbols with standardized meanings; the main graphic symbols are summarized in Table 1.

\subsubsection{Quantitative risk analysis}

The quantification of a fault tree has essentially the aim of quantifying the reliability $R(t)$ of a system, that is the probability that, in correspondence of an allotted time interval (also called mission time, $\mathrm{t}$ ), a system performs properly the function for which it was built. From this definition, it is clear that the reliability of a system is inversely proportional to the mission time: the longer the time of the mission, the smaller results to be the reliability of the system (or it is more likely that the system fails).

It is important to note that the quantification of the fault tree is an operation valid for orders of magnitude; in other words, the results cannot be compared on the basis of small differences. For example, 1.5 and 4.1 are essentially the same number while 3.5 to 0.12 are two numbers significantly different. It follows that no unnecessarily high accuracy is required in the input data (i.e. in the estimate of the probabilities of occurrence of the primary events).

Table 1: Graphic symbols for fault tree analysis.

\begin{tabular}{|c|c|}
\hline Graphic Symbol & Meaning \\
\hline \multirow{5}{*}{ Intermediate Event } & $\begin{array}{l}\text { Primary events: these events, for one reason or another, are } \\
\text { not further investigated; if you want to perform a quantitative } \\
\text { analysis to estimate the frequency of occurrence of the top } \\
\text { event you need to know the frequency of occurrence of these } \\
\text { primary events, for example, estimating it through a historical } \\
\text { analysis. }\end{array}$ \\
\hline & $\begin{array}{l}\text { Intermediate events: these are events that occur before or after } \\
\text { another event and represent the cause of the next event; they }\end{array}$ \\
\hline & $\begin{array}{l}\text { gates. } \\
\text { OR-gate: so that the output of the gate takes place, it is suffi- } \\
\text { cient that one of the inputs to the gate itself (which may be any } \\
\text { number greater than 1) occurs. }\end{array}$ \\
\hline & $\begin{array}{l}\text { AND-gate: so that the output of the gate happens, it is neces- } \\
\text { sary that all the inputs to the gate itself (which may be any } \\
\text { number greater than 1) occur. }\end{array}$ \\
\hline & $\begin{array}{l}\text { INHIBIT-gate: represent a scenario in which the output event } \\
\text { occurs if all input events occur and an additional conditional } \\
\text { event (typically an event external to the configuration repre- } \\
\text { sented by the fault tree) also occurs. }\end{array}$ \\
\hline
\end{tabular}




\subsubsection{Primary events quantification}

For a system whose modes of failure can be represented in a fault tree, the information contained in $R(t)$ are usually summed in the following functions: the unavailability $\mathrm{q}(\mathrm{t})$, which is the probability that the system is not able to perform its function (because it is broken) at time $t$, and the frequency of occurrence $W(t)$, which is the number of times that the system is not expected to be able to perform its function in its mission time.

To quantify a fault tree is first necessary to calculate these quantities for all primary events that have been identified in the construction of the fault tree itself.

If the component involved in the primary event is not repairable, the component is no longer able to perform its function at time $t$ if it is spoiled before the same time $t$; its unavailability therefore coincides with its unreliability, namely [20]:

$$
q(t)=\lambda t
$$

where $\lambda$ is the failure rate, that is, the frequency with which a system fails (or the fraction of the components that fail per unit of time, $\mathrm{y}^{-1}$ ) and $t$ is time, $\mathrm{y}$.

If the component is repairable, its unavailability no longer coincides with its unreliability, since, even if the component had spoiled before time $t$, may still perform its function at time $t$ in the case where it has been repaired. In this case [20], it is possible to demonstrate that the unavailability reaches an asymptotic value equal to:

$$
q(t)=\frac{\lambda T_{D}}{1+\lambda T_{D}} \approx \lambda T_{D}=\frac{\lambda}{\mu}
$$

where $T_{D}$ is the mean repair time, that is the time necessary to repair the component, and $\mu=1 / T_{D}$ is the repair rate, that is, the frequency with which a component can be repaired. For practical purposes, the unavailability can be approximated by the probability that the component has failed in the time interval $T_{D}$ previous than the instant of time considered (therefore, resulting failure at time $t$ ).

\subsubsection{Minimal cut sets and top event quantification [20]}

A Minimal Cut Set (MCS) is the minimum combination of primary events which is necessary and sufficient to ensure the occurrence of the top event; in equivalent terms, the top event occurs if all the events in a MCS occur simultaneously. Then, the unavailability of a MCS is the probability that, at time $t$, all the system components are not able to perform their function; therefore, this probability coincides with the probability of occurrence of the top event due to the considered MCS. Since the individual events involved in a MCS are all independent, the overall probability that all events occur simultaneously is given by the following relation:

$$
q(t)=\prod_{i=1}^{n} q_{i}(t)
$$

where $n$ is the order of the MCS (the number of events that involves the MCS) and $q_{i}(t)$ is the unavailability of each component of the MCS.

The frequency of occurrence of the MCS, which coincides with the frequency of occurrence of the top event in the mission time $(T)$ because of the MCS considered, is then calculated as:

$$
W(t)=\int_{o}^{T}\left[\sum_{i=1}^{n}\left(\left(\prod_{\substack{j=1 \\ j \neq i}}^{n} q_{j}(t)\right) \lambda_{i}\right)\right] d t
$$


A top event can occur if anyone of the MCS that can cause it occurs. The likelihood of the top event in a given interval of time can thus be calculated as the union (logical OR) of the probability of occurrence of each MCS that can cause the top event itself.

\section{CASE STUDY: DE-DUSTING SYSTEM REVAMPING}

In accordance with the purposes of the present work, we have focused on the relevant problem of the triggering of explosions in the de-dusting section of a cement plant. As it is widely known, cement plants can suffer of explosion like problems only in the case of an accidental development of an overpressure inside process equipment. Therefore, much more serious explosions, such as dust explosions, cannot be triggered inside these plants because cement powder cannot burn as it does not contain any combustible group.

Using fault tree analysis, it has been evaluated the changing in the overall risk (considering one year of mission time) of a physical explosion referring to the case study where a hybridlike collector is realized by introducing a Fabric Filter (FF) downstream with respect to an already installed ElectroStatic Precipitator (ESP). This revamping operation is capable of guaranteeing an increase in the de-pulverization efficiency of more than $0.5-1.5 \%$; therefore, it is able to comply with the new environmental laws on dust emissions into the atmosphere.

Thus, we have proceeded with the drafting of the fault trees concerning the top event 'occurrence of an explosion in the de-dusting section' both before (ESP) and after (HYBRID FILTER $=$ ESP + FF) the revamping operation.

For what concerns the layout of the de-dusting section (before and after) and its functioning, reference has been made to the plant sketch reported in Figure 1.

Figure 2 shows the graphical representation of fault tree realized for the plant configuration before the retrofit operation, which is considering the de-dusting section of the plant as constituted by the ESP (and its related instrumentation) only. Observing the tree, it can be derived clearly how an explosion of the ESP can be triggered as a consequence of different chains of events (all intended as failures).

In particular, the top event takes place in the case where both of the following final intermediate events occurs:

- E10: development of an overpressure higher than 6 bar inside the ESP chamber;

- E20: failure of the rupture disk installed on the ESP.

It is important to state that, within the scheme of the fault tree, all the protective devices present into the plant section object of the analysis have been considered.

Performing the MCS analysis, it is possible to observe that there are 4 MCS: 3 of order 4 and 1 of order 5 . Among the MCS of order 4, we have all the combinations of events in which, due to the presence of external factors causing the blockage of the chimney (EXT), the feed to the de-dusting unit (FEED), the absence of a scheduled and correct maintenance activity (MANABS), the blockage of the rupture disk installed on the ESP (SCRBLOC), the absence of a human action as a consequence of the sounding of the high pressure alarm (HE) and the breakdown of the ESP pressure indicator (PI), a pressure overcoming the maximum threshold value can be reached causing a physical explosion of the ESP chamber.

For what concerns the MCS of order 5, it involves the following combination of events: fan breakdown (FAN), absence of a scheduled and correct maintenance activity (MANABS), block of the rupture disk installed on the ESP (SCRBLOC), pressure interlock (PHL) and temperature controller failure (TIC) on the water spray feeding line that constitutes the cooling system available for the hot dusty gases exiting the kiln and addressed to the de-dusting system. 


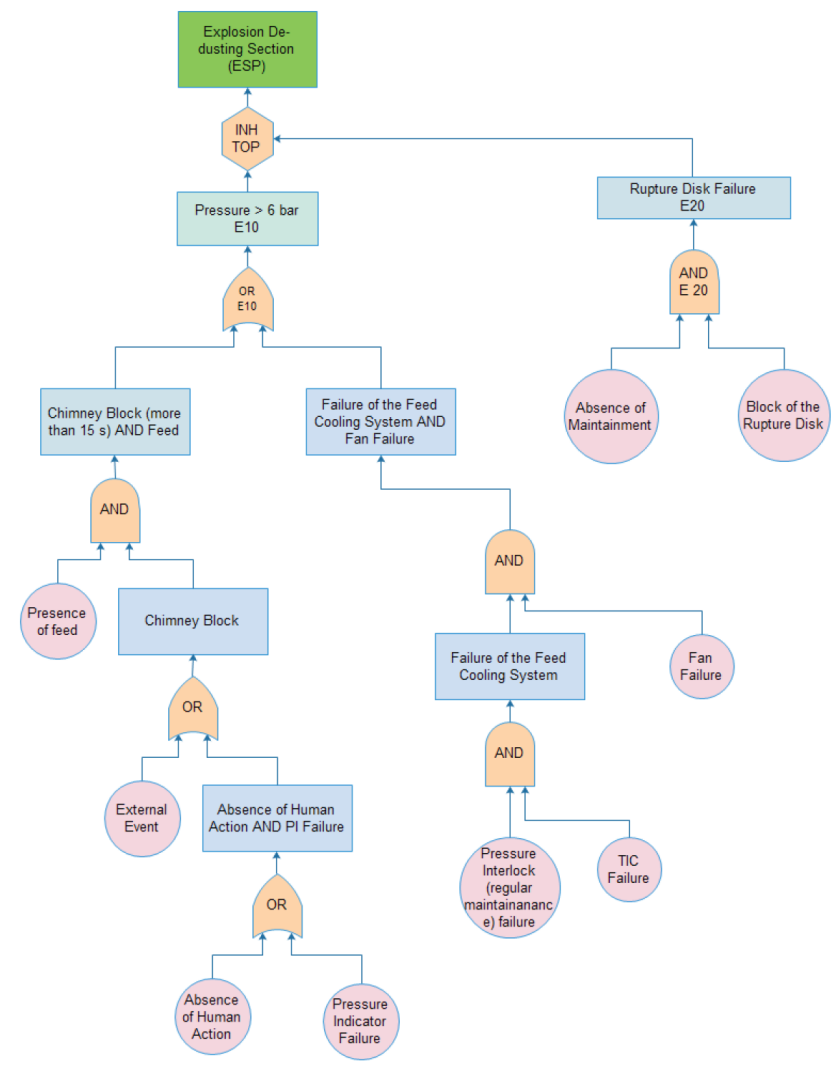

Figure 2: Fault tree for the top event 'explosion in the de-dusting section' evaluated before the plant retrofit.

In order to determine all failure rates and unavailabilities, Dossier Ambiente manuals [21] have been used. Table 2 contains all the data necessary for the quantification of the fault tree.

Quantifying the fault tree using a simple numerical probability calculation involving the computation of all MCS, a value of the probability of occurrence of the top event equal to 5.6 $10^{-8} \mathrm{y}^{-1}$ has been found. Such a value can be located in the acceptability zone for what concern both individual and societal $(<100$ inhabitants) risk. Therefore, just from this analysis, the system does not require to be integrated with redundant protection devices in order to improve the reliability of the de-dusting section.

Successively, we have proceeded with the generation of the fault tree concerning the top event 'occurrence of an explosion in the de-dusting section', now constituted by a fabric filter installed after the exit of the already existing ESP (that is, considering a revamping operation).

In this case, the generated fault tree is too wide to be reported here but it is possible to briefly summarized the main results arising from its detailed analysis.

Particularly, 7 different minimal cut sets can be observed: 3 of order 3, 3 of order 4 and 1 of order 5 .

Among the MCS of order 3, quite dangerous, we have all the combinations of undesired events and failures in which, due to the absence of a regular and correct maintenance activity 
Table 2: Database for failure rates, repair rates and unavailabilities. Case study before the plant revamping.

\begin{tabular}{llll}
\hline & & Repair rate & Unavailability \\
Primary event code & Failure rate $\left[\mathbf{y}^{-\mathbf{1}}\right]$ & {$\left[\mathbf{y}^{-\mathbf{1}}\right]$} & {$[-]$} \\
\hline EXT & - & - & $1 \cdot 10^{-7}$ \\
FEED & - & - & $8 \cdot 10^{-1}$ \\
MANABS & - & - & $2 \cdot 10^{-3}$ \\
SCRBLOC & - & - & $1 \cdot 10^{-2}$ \\
HE & - & - & $2 \cdot 10^{-3}$ \\
PI & $3 \cdot 10^{-1}$ & 200 & $1.5 \cdot 10^{-3}$ \\
FAN & - & - & $1 \cdot 10^{-2}$ \\
PHL & - & - & $1 \cdot 10^{-3}$ \\
TIC & $1 \cdot 10^{-1}$ & 200 & $5 \cdot 10^{-4}$ \\
\hline
\end{tabular}

on the rupture disks of both the fabric filter and the electrostatic precipitator (MANABS), the blockage of the rupture disks (SCRBLOC), the breakdown of the cleaning air timing system (TEMP), the absence of cleaning air (NOAIR) and the failure in closed position of the exiting valve of the fabric filter (VALCLO), a pressure overcoming the maximum threshold value can be reached causing a physical explosion of the hybrid-like collector.

Among the MCS of order 4, we have all the combinations of events previously determined for the ESP unit alone (even if with some little variants): blockage of the chimney (EXT), presence of feed to the de-dusting unit (FEED), absence of maintenance activity (MANABS), block of the installed rupture disks (SCRBLOC), absence of human actions even if a high-pressure alarm is sounding (HE) and breakdown of the hybrid filter differential pressure indicator (PI). Finally, regarding the MCS of order 5, we can observe that it is the same involved in the plant configuration before the retrofit operation (this is because it involves only failures of equipment located upstream of the ESP and the fan).

As previously done, to determine all failure rates and unavailabilities, Dossier Ambiente manuals [21] have been used. Table 3 contains all the data necessary for the quantification of the new fault tree.

From a qualitative point of view, the new criticality that arises from the introduction of a FF downstream to the ESP is a blockage of the gaseous flux inside the FF. This can be due, as the sake of example, to the absence of air into the FF cleaning system: not removed dusts on the filter bags can increase pressure drops inside the equipment so that no gaseous flux can pass anymore. Therefore, the pressure into the FF and, consequently, into the ESP can only increase if there is the failure of the protective systems (rupture disks).

Quantifying the fault tree using a classical numerical probability calculation involving the computation of all MCS, a value of the probability of occurrence of the top event equal to 3.8 $10^{-6} \mathrm{y}^{-1}$ has been found. Such a value is located in the non-acceptability zone for what concern the societal risk ( $<100$ inhabitants) and at the boundary of the acceptability region if an individual risk is considered.

This result confirm that a revamping operation performed without taking into account properly the different operating conditions under which the new revamped plant will operate can be very dangerous. 
Table 3: Database for failure rates, repair rates and unavailabilities. Case study after the plant retrofit.

\begin{tabular}{llll}
\hline & & $\begin{array}{l}\text { Repair rate } \\
{\left[\mathbf{y}^{-\mathbf{1}}\right]}\end{array}$ & $\begin{array}{l}\text { Unavailability } \\
{[-]}\end{array}$ \\
\hline Primary event code & Failure rate $\left[\mathbf{y}^{-\mathbf{1}}\right]$ & - & $1 \cdot 10^{-7}$ \\
FEED & - & - & $8 \cdot 10^{-1}$ \\
MANABS & - & - & $2 \cdot 10^{-3}$ \\
SCRBLOC & - & - & $1 \cdot 10^{-2}$ \\
HE & - & - & $2 \cdot 10^{-3}$ \\
PI & - & 200 & $1.5 \cdot 10^{-3}$ \\
FAN & $3 \cdot 10^{-1}$ & - & $1 \cdot 10^{-2}$ \\
PHL & - & - & $1 \cdot 10^{-3}$ \\
TIC & - & 200 & $5 \cdot 10^{-4}$ \\
NOAIR & $1 \cdot 10^{-1}$ & - & 0.1 \\
TEMP & - & - & 0.05 \\
VALCLO & - & - & 0.05 \\
\hline
\end{tabular}

\section{CONCLUSIONS}

In this paper the frequency of the relevant undesired event 'explosion in the de-dusting section of a cement plant' has been calculated for two different plant configurations: precisely, before and after the performing of an upgrade operation aimed at reducing the atmospheric emission of dusts and particulate matter (addition of a fabric filter downstream an electrostatic precipitator).

Analyzing the fault trees generated for the previously mentioned top event before and after the revamping operation, it has been showed that the probabilities of occurrence considering a mission time equal to 1 year are, respectively, $5.610^{-8} \mathrm{y}^{-1}$ and $3.810^{-6} \mathrm{y}^{-1}$.

Referring to the most standard and widely accepted threshold value for the risk acceptability in industrial areas (which is $1.010^{-6} \mathrm{y}^{-1}$ - reference: individual risk), the risk in the first plant configuration (before the retrofit operation) can be considered acceptable and the system does not require to be integrated with redundant protection devices in order to improve the reliability of the de-dusting section. On the contrary, in the revamped configuration, the risk falls within the non-acceptability zone; therefore, the system requires to be integrated with redundant protection devices in order to improve the reliability of the revamped de-dusting section. Particularly, the critical feature is precisely the introduction of the new apparatus, the fabric filter, which introduces a potential hazard before not present: that is, blocking of the air flux, consequent pressurization of the whole de-dusting line and, finally, explosion.

This simple analysis confirms that each process or pieces of equipment change must be fully evaluated from the safety and reliability viewpoint before being introduced into an already existing plant because it can imply the increase of the risk function. Particularly, in this case, there is an increase of the risk function of about two orders of magnitude: this locates the new configuration of the plant into a non-acceptability risk zone, even if the revamping operation was intended to a better integration of the plant with the environment. 


\section{REFERENCES}

[1] Explosions in Cement Plants. Available at: www.fike.com/

[2] Available at: http://www.greenreport.it/

[3] Rada, E.C., Squazardo, S., Ionescu, G. \& Badea, A., Economic viability of SRF co-combustion in cement factory. UPB Scientific Bulletin, Serie D, 76(3), pp. 199-206, 2014.

[4] Rada, E.C., Ragazzi, M., Ionescu, G., Merler, G., Moedinger, F., Raboni, M. \& Torretta, V., Municipal solid waste treatment by integrated solutions: energy and environmental balances. Energy Procedia, 50, pp. 1037-1044, 2014.

https://doi.org/10.1016/j.egypro.2014.06.123

[5] Copelli, S., Rada, E.C., Ragazzi, M., Torretta V. \& Schiavon, M., Reliability analysis and assessment of the explosion risk in a hybrid collector. WIT Transactions on The Built Environment, 151, pp. 239-250, 2015. https://doi.org/10.2495/safe150211

[6] Torretta, V., Rada, E.C., Panaitescu, V. \& Apostol, T., Some considerations on particulate generated by traffic. UPB Scientific Bulletin, Series D, 74(4), pp. 241-248, 2012.

[7] Pei, B., Cui, H., Liu, H. \& Yan, N. Chemical characteristics of fine particulate matter emitted from commercial cooking. Frontiers of Environmental Science and Engineering, 10(3), pp. 559-568, 2016. https://doi.org/10.1007/s11783-016-0829-y

[8] Fuller, G.W., Tremper, A.H., Baker, T.D., Yttri, K.E. \& Butterfield, D., Contribution of wood burning to PM10 in London. Atmospheric Environment, 87, pp. 87-94, 2014. https://doi.org/10.1016/j.atmosenv.2013.12.037

[9] Gutierrez Ortiz, F.J., Navarrete, B., Caňadas, L. \& Salvador, L., A technical assessment of a particle hybrid collector in a pilot plant. Chemical Engineering Journal, 127, pp. 131-142, 2007.

https://doi.org/10.1016/j.cej.2006.09.015

[10] Long, Z. \& Yao, O., Numerical simulation of the flow and the collection mechanism inside a scale hybrid particulate collector. Powder Technology, 215-216, pp. 26-37, 2012.

https://doi.org/10.1016/j.powtec.2011.08.045

[11] Böttner, C.U., The role of the space charge density in particulate processes in the example of the electrostatic precipitator. Powder Technology, 135-136, pp. 285-294, 2003. https://doi.org/10.1016/j.powtec.2003.08.020

[12] Neimarlija, N., Demirdžić, I. \& Mrzaferija, S., Finite volume method for calculation of electrostatic fields in electrostatic precipitators. Journal of Electrostatics, 67, pp. 37-47, 2009.

[13] Cooperman, G., A unified efficiency theory for electrostatic precipitator. Atmospheric Environment, 18, pp. 277-285, 1984. https://doi.org/10.1016/j.elstat.2008.10.007

[14] Varonos, A.A., Anagnostopoulos, J.S. \& Bergeles, G.C., Prediction of the cleaning efficiency of an electrostatic precipitator. Journal of Electrostatics, 55, pp. 111-133, 2002. https://doi.org/10.1016/s0304-3886(01)00187-5

[15] Piccinini, N. \& Demichela, M., Risk based decision-making in plant design. Canadian Journal of Chemical Engineering, 86, pp. 316-322, 2008.

https://doi.org/10.1002/cjce.20064 
[16] Tixier, J., Dusserre, G., Salvi, O. \& Gaston, D., Review of 62 risk analysis methodologies of industrial plants. Journal of Loss Prevention in the Process Industries, 15, pp. 291-303, 2002. https://doi.org/10.1016/s0950-4230(02)00008-6

[17] Piccinini, N. \& Ciarambino, I., Operability analysis de-voted to the development of logic trees. Reliability Engineering \& System Safety, 55, pp. 227-241, 1997. https://doi.org/10.1016/s0951-8320(96)00111-1

[18] CCPS, Guidelines for Chemical Process Quantitative Risk Analysis, Wiley, 2nd ed., 1999.

[19] Vaseley, W.E., Goldberg, F.F., Roberts, N.H. \& Haasl, D.F., Fault Tree Handbook, U.S. Nuclear Regulatory Commission Washington D.C., 1981.

[20] Rota, R. \& Nano, G., Introduzione alla affidabilità e sicurezza nell'industria di processo, Pitagora Editrice, 2007.

[21] Dossier Ambiente, http://www.amblav.it/dossier.aspx 